\title{
Establishment and characterization of a chordoma cell line from the tissue of a patient with dedifferentiated-type chordoma
}

\author{
Jeong-Yub Kim, MS, ${ }^{1,4}$ Jongsun Lee, MS, ${ }^{1}$ Jae-Soo Koh, MD, ${ }^{2}$ Myung-Jin Park, PhD, ${ }^{1}$ and \\ Ung-Kyu Chang, MD ${ }^{3}$
}

\begin{abstract}
'Division of Radiation Cancer Research, Research Center for Radio-Senescence, ${ }^{2}$ Department of Pathology, and ${ }^{3}$ Department of Neurosurgery, Korea Institute of Radiological and Medical Sciences; and ${ }^{4}$ Department of Pathology, College of Medicine, Korea University, Seoul, South Korea
\end{abstract}

\begin{abstract}
OBJECTIVE Chordoma is a rare bone tumor of the axial skeleton believed to originate from the remnants of the embryonic notochord. The available tumor cells are characteristically physaliferous and express brachyury, a transcription factor critical for mesoderm specification. Although chordomas are histologically not malignant, treatments remain challenging because they are resistant to radiation therapy and because wide resection is impossible in most cases. Therefore, a better understanding of the biology of chordomas using established cell lines may lead to the advancement of effective treatment strategies. The authors undertook a study to obtain this insight.
\end{abstract}

METHODS Chordoma cells were isolated from the tissue of a patient with dedifferentiated-type chordoma (DTC) that had recurred. Cells were cultured with DMEM/F12 containing 10\% fetal bovine serum and antibiotics (penicillin and streptomycin). Cell proliferation rate was measured by MTS assay. Cell-cycle distribution and cell surface expression of proteins were analyzed by fluorescence-activated cell sorting (FACS) analysis. Expression of proteins was analyzed by Western blot and immunocytochemistry. Radiation resistance was measured by clonogenic survival assay. Tumor formation was examined by injection of chordoma cells at hindlimb of nude mice.

RESULTS The putative (DTC) cells were polygonal and did not have the conventional physaliferous characteristic seen in the U-CH1 cell line. The DTC cells exhibited similar growth rate and cell-cycle distribution, but they exhibited higher clonogenic activity in soft agar than U-CH1 cells. The DTC cells expressed high levels of platelet-derived growth factor receptor- $\beta$ and a low level of brachyury and cytokeratins; they showed higher expression of stemness-related and epithelial to mesenchymal transition-related proteins than the U-CH1 cells. Intriguingly, FACS analysis revealed that DTC cells exhibited marginal surface expression of CD24 and CD44 and high surface expression of CXCR4 in comparison to U-CH1 cells. In addition, blockade of CXCR4 with its antagonist AMD3100 effectively suppressed the growth of both cell lines. The DTC cells were more resistant to paclitaxel, cisplatin, etoposide, and ionizing radiation than the U-CH1 cells. Injection of DTC cells into the hindlimb region of nude mice resulted in the efficient formation of tumors, and the histology of xenograft tumors was very similar to that of the original patient tumor.

CONCLUSIONS The use of the established DTC cells along with preestablished cell lines of chordoma may help bring about greater understanding of the mechanisms underlying the chordoma that will lead to therapeutic strategies targeting chordomas.

http://thejns.org/doi/abs/10.3171/2016.3.SPINE151077

KEY WORDS chordoma; dedifferentiated-type chordoma; brachyury; CXCR4; oncology

ABBREVIATIONS DTC = dedifferentiated-type chordoma; EGFR = epidermal growth factor receptor; EMT $=$ epithelial to mesenchymal transition; FACS = fluorescenceactivated cell sorting; FBS = fetal bovine serum; IR = ionizing radiation; $p$ - = phosphorylated; PBS = phosphate-buffered saline; PDGFR = platelet-derived growth factor receptor; PTEN = phosphatase and tensin homolog; RT = room temperature; TBST = Tris-buffered saline with Tween 20.

SUBMITTED September 10, 2015. ACCEPTED March 4, 2016.

INCLUDE WHEN CITING Published online June 17, 2016; DOI: 10.3171/2016.3.SPINE151077. 
$\mathrm{C}$ HORDOMAS are rare, slow-growing bone tumors that mostly arise from remnants of the notochord. ${ }^{7,13}$ Histologically, chordomas are classified as conventional (classic), chondroid, and dedifferentiated type. ${ }^{4}$ Although rare, these tumors can be life-threatening due to their local aggressiveness, invasiveness, and progressive natures. The standard treatment for chordoma is adjuvant radiotherapy after excision. However, this current treatment is greatly ineffective possibly because of the local invasiveness followed by recurrence. ${ }^{4}$

Because research in this field is limited, the establishment of various types of chordoma cell lines would be a beneficial tool to move the research forward. Although several studies have reported the establishment of chordoma cell lines, 3,5,9,12,15-18,21 only a few have been validated. In addition, among the validated chordoma cell lines, only a few were reported to be useful as xenograft models., ${ }^{5,12,16}$ For example, in the NOD/SCID/interleukin 2 receptor [IL2r] $\gamma^{\text {null }}$ mouse model, the U-CH1 cell line was able to produce a xenograft tumor whose morphological features and immunohistochemical staining features resembled typical conventional-type chordoma. ${ }^{16}$ However, a dedifferentiated-type chordoma (DTC) cell line has yet to be established. Therefore, more defined chordoma cell lines capable of forming tumors in mice would be a valuable resource for understanding the biology of the tumor and to identify therapeutic targets.

In this study, we attempted to establish a chordoma cell line from human tissue obtained in a patient with a DTC, characterizing the cell line in vitro and in vivo by comparing it to the preexisting conventional-type chordoma cell line, U-CH1.

\section{Methods}

\section{Clinical Presentation}

A 48-year-old female patient developed pain in her coccyx and radiating pain in her lower extremities. Initial MRI showed a well-enhancing mass of the S2-4 along with sacral nerve root compression. The tumor was resected from the sacral region, and sacral nerve root decompression was performed. Postoperative histological diagnosis confirmed that the tumor was a DTC that was composed of mainly typical conventional chordoma areas, with predominantly epithelioid features and exhibiting occasional physaliferous cells and a focal high-grade sarcomatous area (Fig. 1A). After surgery and recovery, the patient was discharged from the hospital with relief of her preoperative symptoms. However, 4 months later, she returned to our clinic, suffering from right leg pain and weakness. MRI revealed a recurrent tumor mass at the S-1 vertebral body (Fig. 1B). A signal indicative of a recent bleed was observed. A second operation was performed, and the pathological diagnosis was DTC exhibiting ovoid to spindle highly malignant sarcomatous tumor cells (Fig. 1C). Conventional chordoma-like areas were not identified in this time. This indicates an aggressive transformation has occurred. Tumor tissue from the second operation was used to develop a primary cell line.

\section{Reagents}

Antibodies against platelet-derived growth factor receptor (PDGFR) $-\alpha$, PDGFR- $\beta$, cytokeratin $8 / 18$, brachyury, c-Met, CD24, CD44, Oct4, ZEB1, CD133, c-Myc, and $\beta$-actin were purchased from Santa Cruz Biotechnology. Antibodies against phosphorylated (p)-Akt (T308 and S473), p-STAT3 (Y705 and S727), p-ERK1/2, p-JNK, p-mTOR, Snail, Slug, phosphatase and tensin homolog (PTEN), and Musashi were obtained from Cell Signaling Biotechnology. Sox 2 was purchased from R\&D Systems. Antibodies against epidermal growth factor receptor (EGFR), E-cadherin, and N-cadherin were obtained from BD Biosciences (Becton Dickinson). Antibodies against Nestin, Notch1, and Nanog were acquired from Millipore. Antibodies against Twist, FoxM1, and CXCR4 were obtained from Abcam. Vimentin was purchased from Thermo Scientific and growth factor-reduced Matrigel was purchased from BD Biosciences.

\section{Cell Culture}

Human primary chordoma cells were obtained from a fresh specimen with the approval of institutional review board as a part of the study protocol. The chordoma specimen was cut into fine pieces with a scalpel and subsequently cultured in DMEM/F12 (Cellgro) supplemented with $2 \mathrm{mM}$ glutamine, $1 \mathrm{mM}$ sodium pyruvate, $100 \mathrm{U} /$
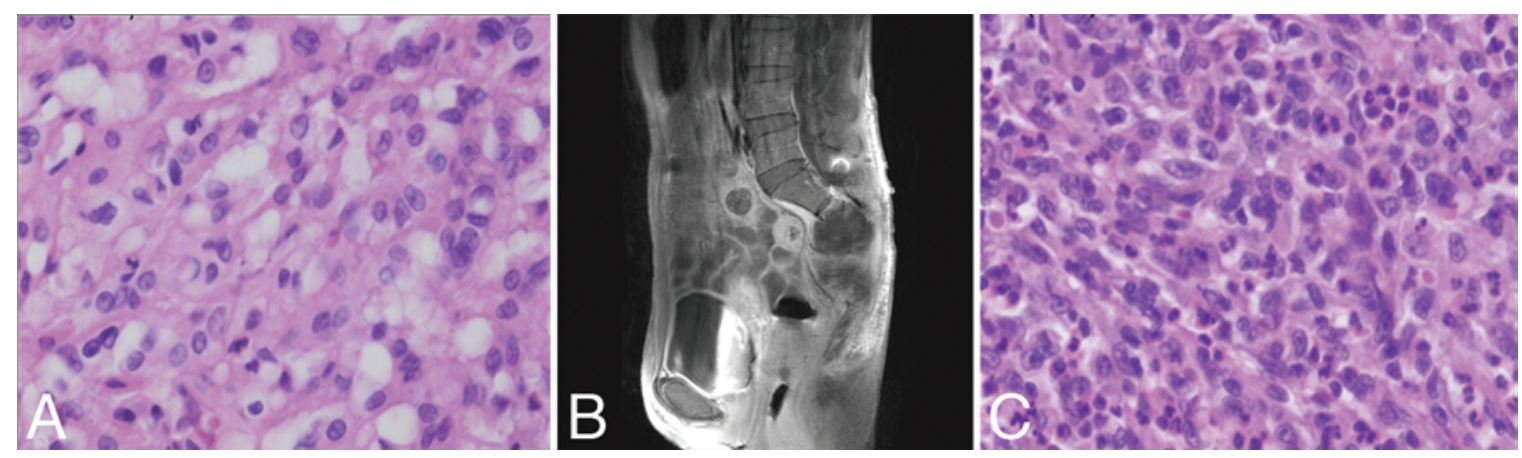

FIG. 1. A: Pathological findings of the first operation: low-grade epithelioid tumor cells with abundant eosinophilic cytoplasm and occasional cytoplasmic vacuoles. $\mathrm{H} \& \mathrm{E}$, original magnification $\times 400$. B: MRI finding at the presentation with recurrent tumor; the mass involves the proximal resection margin at the S-2 vertebral body. C: Pathological findings of the second operation: highgrade sarcomatous feature with hypercellularity and severe atypism. $\mathrm{H} \& \mathrm{E}$, original magnification $\times 400$. Figure is available in color online only. 
$\mathrm{ml}$ penicillin, $100 \mu \mathrm{g} / \mathrm{ml}$ streptomycin (Gibco), and $10 \%$ heat-inactivated fetal bovine serum (FBS; Gibco) in a humidified incubator containing $5 \% \mathrm{CO}_{2}$ at $37^{\circ} \mathrm{C}$. The established chordoma cell line $\mathrm{U}-\mathrm{CH} 1^{18}$ was provided by the Chordoma Foundation (Durham, NC) and cultured in Iscove/RPMI (4:1) medium containing 10\% FBS, 2 mM glutamate, and antibiotics as described above.

\section{MTS (3-(4,5-Dimethylthiazol-2-yl)-5-(3- Carboxymethoxyphenyl)-2-(4-Sulfophenyl)-2H- Tetrazolium) Assays}

Cells were seeded in 96-well $\left(5 \times 10^{3}\right.$ cells/well $)$ plates and incubated at $37^{\circ} \mathrm{C}$ under $5 \% \mathrm{CO}_{2}$ incubator for 24 hours. The medium was replaced with $100 \mu \mathrm{l}$ of complete medium with or without various drugs (cisplatin, paclitaxel, etoposide, AMD3100, AMD3465 [Sigma-Aldrich]) and ionizing radiation (IR) at the indicated concentrations and doses, respectively. After 48 hours of incubation for drugs and 96 hours of incubation for IR exposure, wells were replaced with fresh medium $(100 \mu \mathrm{l} /$ well $)$ with the addition of $20 \mu \mathrm{l}$ of MTS solution (CellTiter 96 Aqueous MTS Reagent Powder) and $1 \mu \mathrm{l}$ of phenazine methosulfate in a each well, followed by incubation for 2 hours at $37^{\circ} \mathrm{C}$. After incubation, optical density was measured at $490 \mathrm{~nm}$ using plate reader (BioRad).

\section{Immunocytochemistry}

Chordoma cells were fixed with $4 \%$ paraformaldehyde with $0.1 \%$ Triton $\mathrm{X}-100$ for 10 mins at room temperature (RT). After washing with phosphate-buffered saline (PBS) 3 times for 5 mins each, cells were blocked with blocking solution (5\% bovine serum albumin and $0.5 \%$ Triton X-100 in PBS) for 1 hour at RT, stained with primary antibodies in blocking solution (1:100) for 2 hours, and washed 3 times with PBS. Staining was visualized using Alexa Fluor 488 goat anti-rabbit (A11008) and Alexa Fluor 594 goat anti-mouse (A11005) (Invitrogen) secondary antibodies (1:1000) in dark condition for 1 hour. Nuclei were stained using DAPI (containing mounting solution), and stained cells were viewed under a confocal laser scanning microscope.

\section{Western Blot Analysis}

Chordoma cells were lysed in lysis buffer $(20 \mathrm{mM}$ Tris, $\mathrm{pH}$ 7.4, $150 \mathrm{mM} \mathrm{NaCl}, 1 \mathrm{mM}$ EDTA, $1 \mathrm{mM}$ EGTA, $1 \%$ Triton, $2.5 \mathrm{mM}$ sodium pyrophosphate, $1 \mathrm{mM} \beta$-glycerolphosphate, $1 \mathrm{mM} \mathrm{Na} \mathrm{VO}_{4}, 1 \mu \mathrm{g} / \mathrm{ml}$ leupeptin, and 1 $\mathrm{mM}$ PMSF). The lysates were clarified by centrifugation at $12,000 \mathrm{~g}$ for $10 \mathrm{mins}$ at $4{ }^{\circ} \mathrm{C}$, and protein content in the supernatant was measured by Bradford's method. An aliquot (30-50 $\mu$ g protein per lane) of the total protein was separated by $10 \%$ or $12 \%$ SDS-PAGE and blotted to nitrocellulose transfer membrane $(0.2 \mu \mathrm{m}$; Amersham). The membrane was blocked with $5 \%$ nonfat skim milk in Tris-buffered saline with Tween 20 (TBST) $(20 \mathrm{mM}$ Tris-HCl, pH 7.6, $137 \mathrm{mM} \mathrm{NaCl}$, and $0.01 \%$ Tween 20) for 1 hour at RT, followed by overnight incubation with the primary antibodies at $4^{\circ} \mathrm{C}$. After extensive washing with TBST, the membrane was re-probed with horseradish peroxidase-linked anti-rabbit immunoglobulin, at 1:1000 diluted in 5\% nonfat skim milk in TBST, for 1 hour at RT. Immunoblots were visualized by enhanced chemiluminescence (Amersham) according to the manufacturer's protocol.

\section{Fluorescence-Activated Cell Sorting (FACS) Analysis}

Cells were dissociated into single cells and were washed with PBS followed by stained with phycoerythrinconjugated antibodies at a $4^{\circ} \mathrm{C}$ refrigerator for 30 mins. After washing with PBS twice, cells were analyzed on FACSCalibur (Becton Dickinson).

\section{Soft-Agar Clonogenic Assays}

For soft-agar clonogenic assay, single-cell suspensions $\left(5 \times 10^{3}\right.$ cells $\left./ \mathrm{ml}\right)$ were obtained in $2 \times$ DMEM/F12 with serum $(10 \%)$, resuspended in the same volume of $0.7 \%$ low-melting agar (final $0.35 \%$ ), and poured onto 24-well plates coated at the bottom with agar (1:1 mixture of $2 \times$ DMEM/F12 and 1\% low-melting agar; final 0.5\%). After 14 days of incubation, colonies in 5 random fields per well were counted under a microscope.

\section{Ionizing Radiation Exposure}

For measuring IR sensitivity, cells were seeded in 60$\mathrm{mm}$ dishes $\left(5 \times 10^{2}\right)$ and were exposed to $\gamma$-rays from a ${ }^{137} \mathrm{Cs} \gamma$-ray source (Atomic Energy of Canada, Korea Institute of Radiological and Medical Sciences) at a dose rate of $3.81 \mathrm{~Gy} / \mathrm{min}$.

\section{Immunohistochemistry}

The paraffin sections of xenograft tumor tissues were dewaxed in xylene for 20 mins, followed by sequential hydration in $100 \%, 95 \%, 90 \%$, and $80 \%$ ethanol solutions. After rinsing with PBS, endogenous peroxidase activity was blocked by $3 \%$ hydrogen peroxide treatment for 30 mins. Primary antibodies against cytokeratin (1:1000, Biogenex) and CXCR4 (1:2000, Abcam) were applied to the sections overnight in a moisture chamber at $4^{\circ} \mathrm{C}$. After rinsing with PBS, the sections were incubated with secondary antibody for 10 mins at RT, rinsed with PBS, and incubated with horseradish peroxide-conjugated tertiary antibody for 10 mins at RT. After rinsed with PBS, sections were incubated with diaminobenzidine for 10 mins, counterstained with Meyer's hematoxylin, dehydrated, and mounted.

\section{In Vivo Tumor Formation}

All animal protocols and studies were conducted in accordance with the guidelines of the Institutional Animal Care and Use Committee in the Korea Institute of Radiological and Medical Sciences. Nude mice (female, 15-18 g, 6 weeks old) were obtained from Nara Biotech Co. DTC cells $\left(5 \times 10^{5}\right.$ cells) were mixed with Matrigel (1:1 mixture) and injected into the left flanks of the mice. Tumor size was measured every 2-3 days when the tumor volume reached $50 \mathrm{~mm}^{3}$.

\section{Results}

\section{Primary Culture of Chordoma Cells}

First, we tried to isolate chordoma cells from a recur- 
A
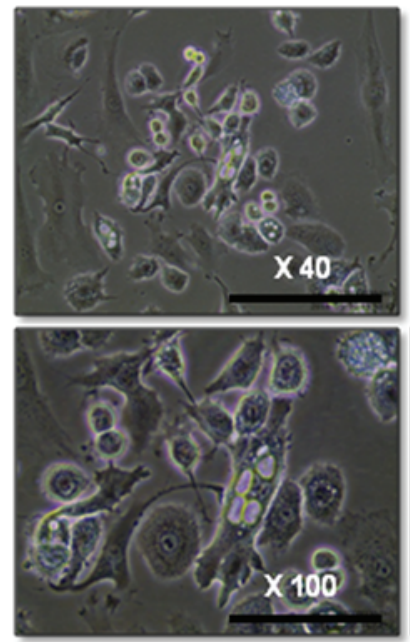

C
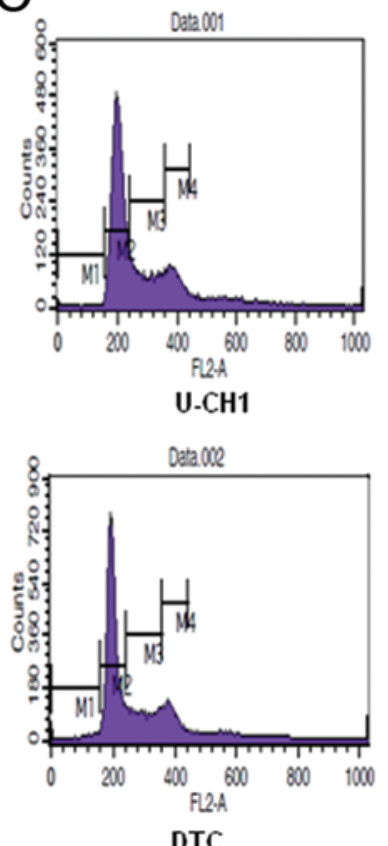

DTC
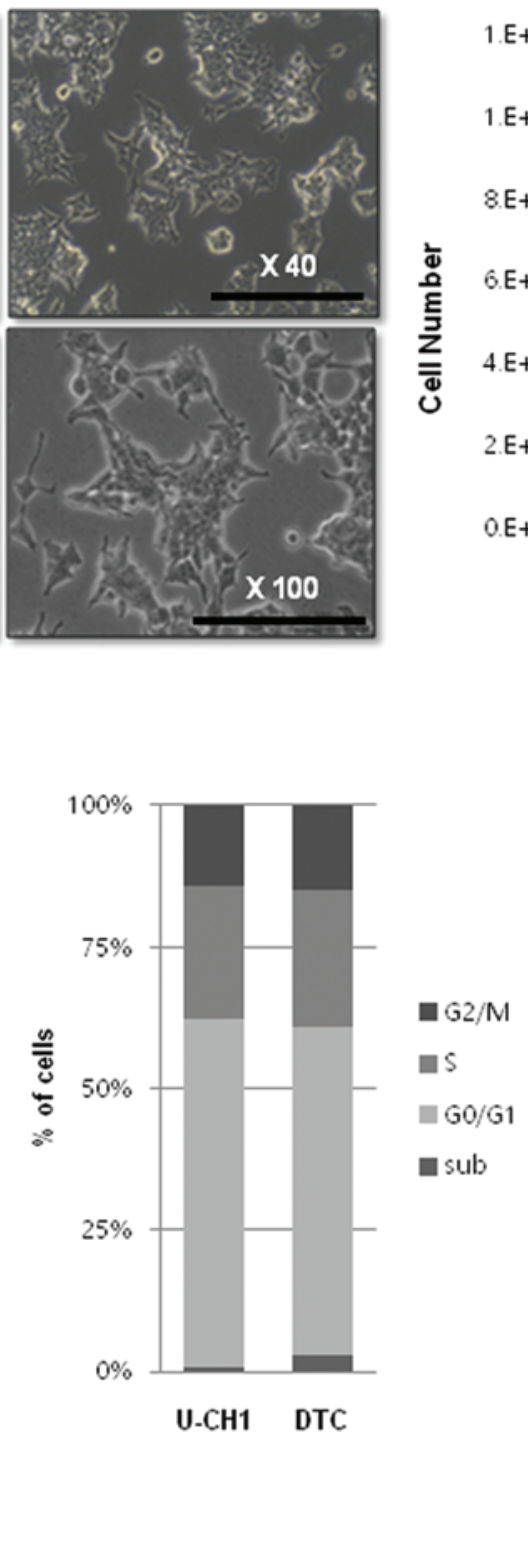

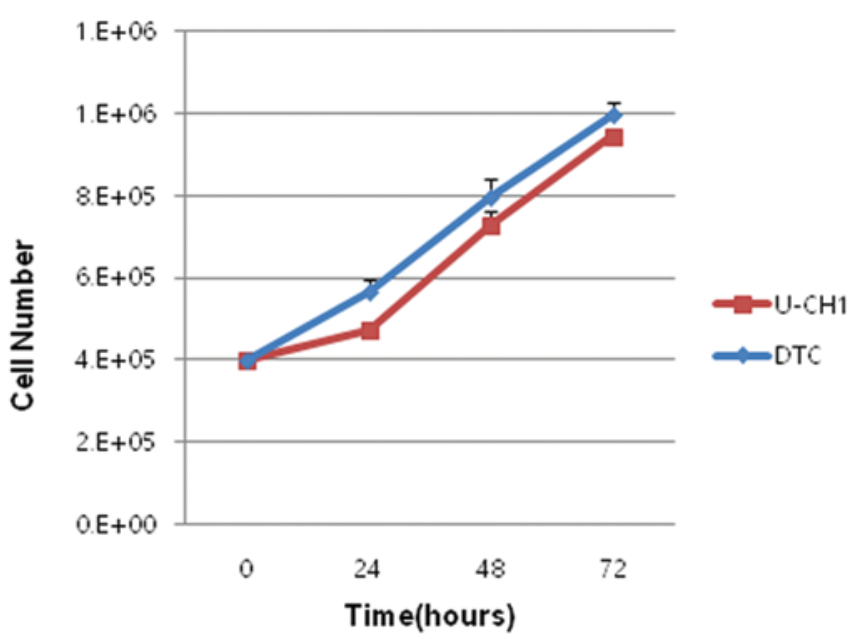

D<smiles>C1CCCC1</smiles>
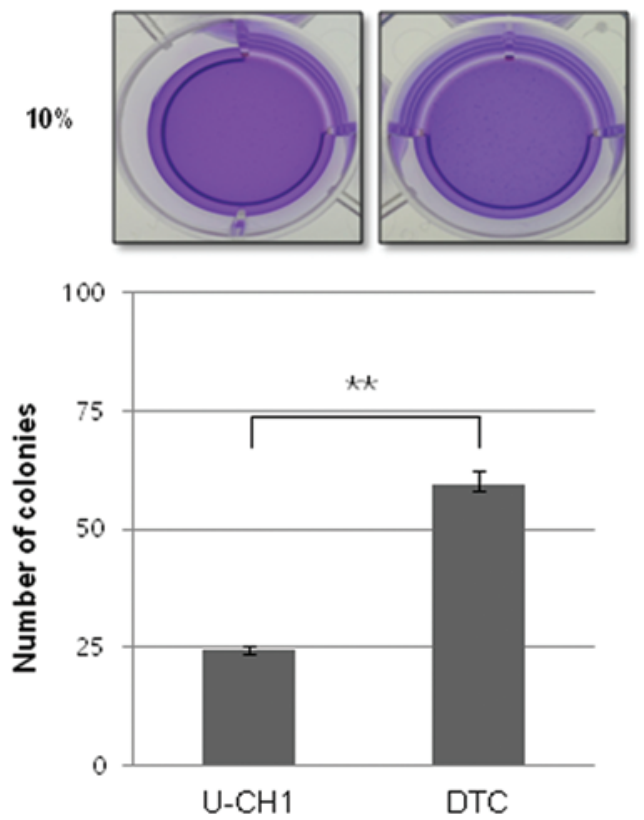

FIG. 2. Cultural characteristics of DTC and U-CH1 cells. A: Morphology of DTC and U-CH1 cells. Original magnification $\times 40$ (upper) and $\times 100$ (lower). B: MTS assay of DTC and U-CH1 cells for measuring cell proliferation rate. C: Cell-cycle analysis of DTC and U-CH1 cells to detect cell-cycle distribution. Representative histogram (left) and quantification of the analysis (right). D: Softagar clonogenic assay of DTC and U-CH1 cells. ${ }^{* *} 0.005<p<0.01$. Figure is available in color online only.

rent tumor specimen from the patient with DTC. The aseptically delivered fresh chordoma specimen from the operating room was dissected using a scalpel and cultured in tissue culture flasks in DMEM/F12 media containing $10 \%$ FBS in a humidified chamber at $37^{\circ} \mathrm{C}$ with $5 \% \mathrm{CO}_{2}$ conditions. After 3 weeks of culture, chordoma cells were visualized under a phase-contrast microscope and were seen to form colonies with spikelike edges. This characteristic is not typical morphology of the reported chordoma cell line $\mathrm{U}-\mathrm{CH} 1$, in which many vacuoles are present (Fig. 2A). ${ }^{18,21}$ MTS assays revealed that the established DTC cells ex- hibited similar proliferation as U-CH1 cells (Fig. 2B). FACS analysis indicated that there was no significant difference in the cell-cycle progression between the two cell lines (Fig. 2C). In soft-agar clonogenic assays, a surrogate method for measuring tumorigenic potential in vitro, DTC cells showed much higher potential for clonogenic activity than did the U-CH1 cells (Fig. 2D). Taken together, these data suggest that the primary-cultured DTC cell line has a similar proliferation capacity and higher in vitro tumorigenic potential than the conventional-type chordoma cell line, U-CH1. 


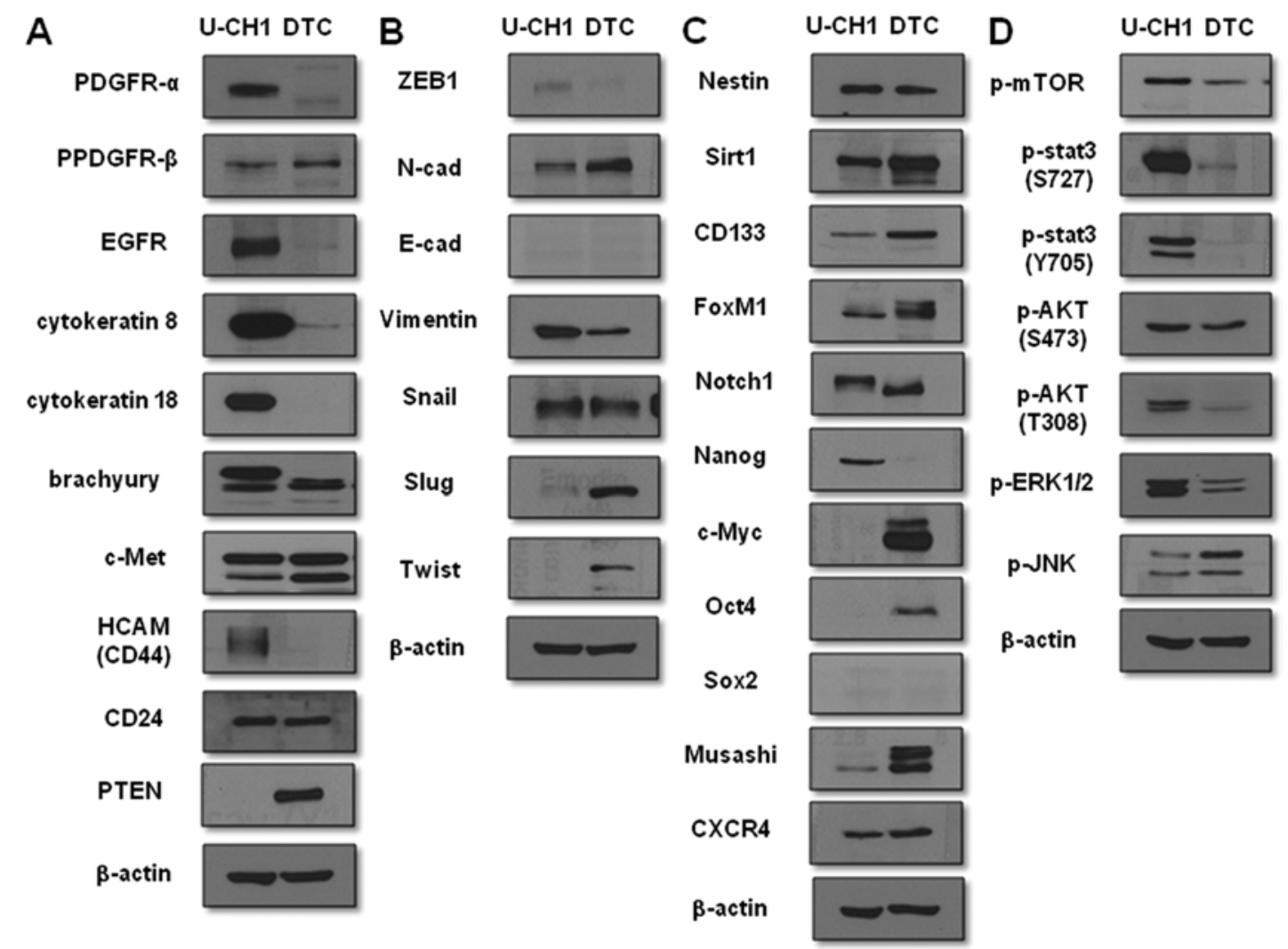

FIG. 3. Molecular expression patterns of DTC and U-CH1 cells. A-D: Western blot analysis of DTC and U-CH1 cells. Total cells were lysed in lysis buffer and subjected to Western blot using antibodies. $\beta$-actin was used as a loading control.

\section{Different Molecular Expression Patterns Between DTC and UCH-1 Cells}

Next we examined molecular expression patterns of DTC cells and compared them to those of the U-CH1 cells. In the chordoma-related gene expression patterns, DTC cells showed higher expression of PDGFR- $\beta$ and lower expression of PDGFR- $\alpha$, EGFR, cytokeratin 8/18, CD44, and brachyury than U-CH1 cells (Fig. 3A). c-Met and CD24 expression were similar between the two cell lines. PTEN, a tumor suppressor protein, was expressed in DTC cells; however, as described in a previous study, the U-CH1 cells did not express PTEN. ${ }^{3}$ Reduced expression of cytokeratin 8/18 is an indication of the epithelial to mesenchymal transition (EMT), which was investigated in these cells. As shown in Fig. 3B, DTC cells showed significantly higher expression of EMT factors including N-cadherin, Slug, and Twist than did U-CH1 cells; Snail expression was only marginally lower in the DTC cells. E-cadherin expression was very weak in both cell lines; however, vimentin expression was higher in the $\mathrm{U}-\mathrm{CH} 1$ cells than in the DTC cells. Intriguingly, DTC cells exhibited enhanced expression of stemness-related molecules including CD133, c-Myc, Oct4, Sirt1, FoxM1, and Musashi, whereas the expression of nestin, Sox2, and Notch1 was not enhanced (Fig. 3C). CXCR4 was slightly upregulated in DTC cells; however, Nanog expression was much lower than that in the U-CH1 cells (Fig. 3C). With regard to the activation of cellular signaling molecules, DTC cells exhibited enhanced expression of p-JNK and reduced expression of p-STAT3 (S727, Y705), p-Akt (S473, T308), p-ERK1/2, and p-mTOR (Fig. 3D). Immunohistochemical staining confirmed the expression pattern of the critical molecules in both cell lines (Fig. 4). Interestingly, PDGFR- $\beta$ and CXCR4 stained blurrylike patterns in DTC cells, indicating the membranous and cytosolic staining of these molecules. FACS analysis was performed to examine the membrane expression of these molecules. The results indicated that DTC cells have a high level of cell surface expression of CXCR4, but not PDGFR- $\beta$ (Fig. 5). In contrast, U-CH1 cells showed a higher level of CD24 and CD44-cell surface glycoproteins that are used for prognostic markers in chordo$\mathrm{ma}^{8,11,19}$ - expression on their cell surfaces than DTC cells (Fig. 5). The molecular characteristics of various chordoma cell lines are summarized in Table 1.

\section{Sensitivity of DTC Cells to Different Drugs and IR}

We next compared the sensitivity to drugs and IR between DTC and U-CH1 cells. Treatment with paclitaxel, cisplatin, and etoposide reduced cell viability in a dosedependent manner; however, the DTC cells were more resistant to these drugs than U-CH1 cells (Fig. 6). Because cell surface expression of CXCR4 is enhanced in DTC cells, we investigated the effect of the CXCR4 antagonist AMD3100 on the proliferation of DTC and U-CH1 cells. As shown in the lower panel of Fig. 6, AMD3100 suppressed the proliferation of both cell lines, but U-CH1 cells were more susceptible to this drug than the DTC 


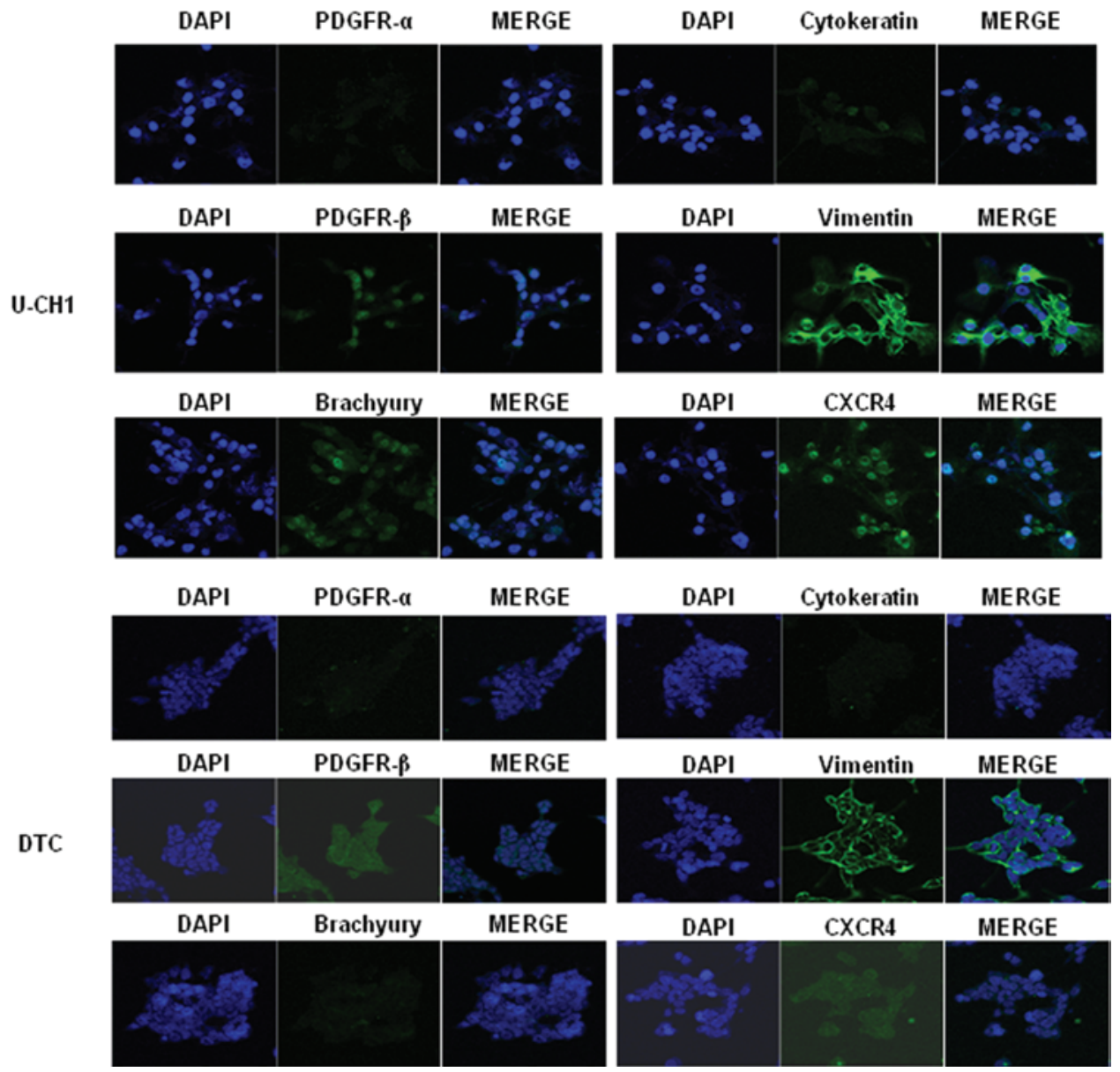

FIG. 4. Immunocytochemistry of DTC and U-CH1 cells with antibodies. Immunofluorescence staining was visualized by probing with Alexa Fluor 594 goat anti-mouse secondary antibody (green). Nuclei were visualized by DAPI staining (blue). Original magnification $\times 100$. Figure is available in color online only.
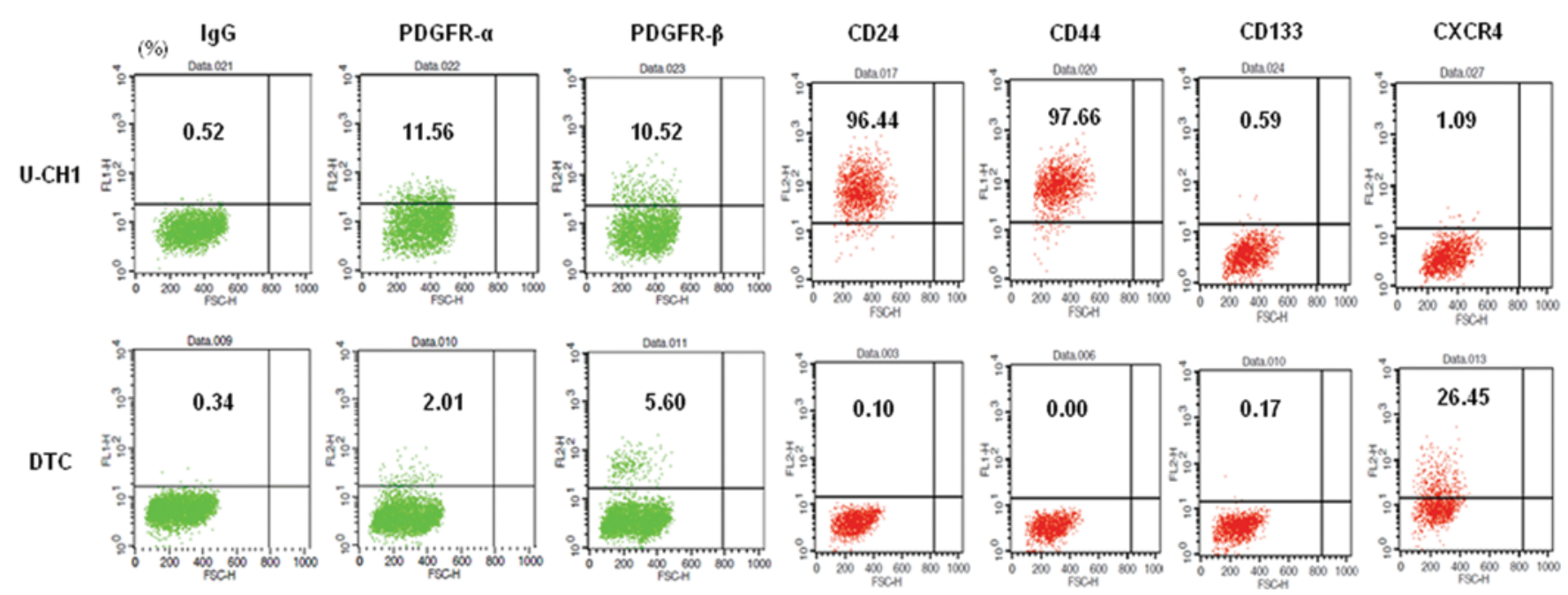

FIG. 5. FACS analysis of DTC and U-CH1 cells for evaluating cell surface expression of the proteins with specific antibodies. Figure is available in color online only. 
TABLE 1. Comparison of expression pattern of chordoma-related proteins in various chordoma cell lines

\begin{tabular}{|c|c|c|c|c|c|c|c|}
\hline \multirow[b]{2}{*}{ Cell Line } & \multicolumn{5}{|c|}{ Protein Expression Pattern } & \multirow[b]{2}{*}{ Chordoma Type } & \multirow[b]{2}{*}{ Reference No.* } \\
\hline & Brachyury & Cytokeratin & CD24 (cell surface) & PTEN & CXCR4 (cell surface) & & \\
\hline $\mathrm{U}-\mathrm{CH} 1$ & ++ & ++ & ++ & - & - & Conventional & $5,10,11,13$ \\
\hline $\mathrm{U}-\mathrm{CH} 2$ & ++ & ++ & ++ & + & ND & Conventional & 12,13 \\
\hline MUG-Chor1 & ++ & ++ & ++ & - & ND & Conventional & 9 \\
\hline $\mathrm{CH} 22$ & ++ & ++ & ++ & ND & ND & Conventional & 10 \\
\hline $\mathrm{JHC7}$ & ++ & ++ & ND & ND & ND & Conventional & 6 \\
\hline $\mathrm{EACH}-1$ & ++ & + & ND & ND & ND & Conventional & 8 \\
\hline DTC & + & + & - & + & ++ & Dedifferentiated & Present study \\
\hline
\end{tabular}

$\mathrm{ND}=$ not determined; $++=$ strong; $+=$ weak; $-=$ none .

* See reference list for corresponding authors and study title.

cells. Another CXCR4 antagonist, AMD3465, also inhibited the proliferation of the DTC and U-CH1 cells but its activity was much weaker than AMD3100 (Fig. 6). Finally, to evaluate the IR sensitivity of both cell lines, we performed MTS-based assays and found that the DTC cells exhibited greater survival activity after IR exposure than the U-CH cells (Fig. 7). These data suggest that the DTC cells exhibited greater resistance to drugs and IR exposure than the U-CH1 cells.

\section{In Vivo Tumor Formation of DTC Cells}

Next, we examined the tumor formation of the DTC cells in vivo. Injection of $5 \times 10^{5}$ DTC cells in the flank region of mice was sufficient to induce tumor formation within 2 weeks of the injection in all 6 mice that were injected with the cells (Fig. 8A). An exponential growth pattern of xenograft tumor is shown in Fig. 8B. Histological examination indicated that mouse xenograft tumor exhibited a similar morphology to that of the patient's tissue, with slightly decreased cytokeratin and CXCR4 expression (Fig. 8C). The data indicate that primary-cultured DTC cells would be useful to develop effective therapeutic approaches to treat patients with DTCs.

\section{Discussion}

Because of the limited availability of chordoma cell lines, it is critical to establish defined cell lines from various chordoma tissues. This advancement will allow for a
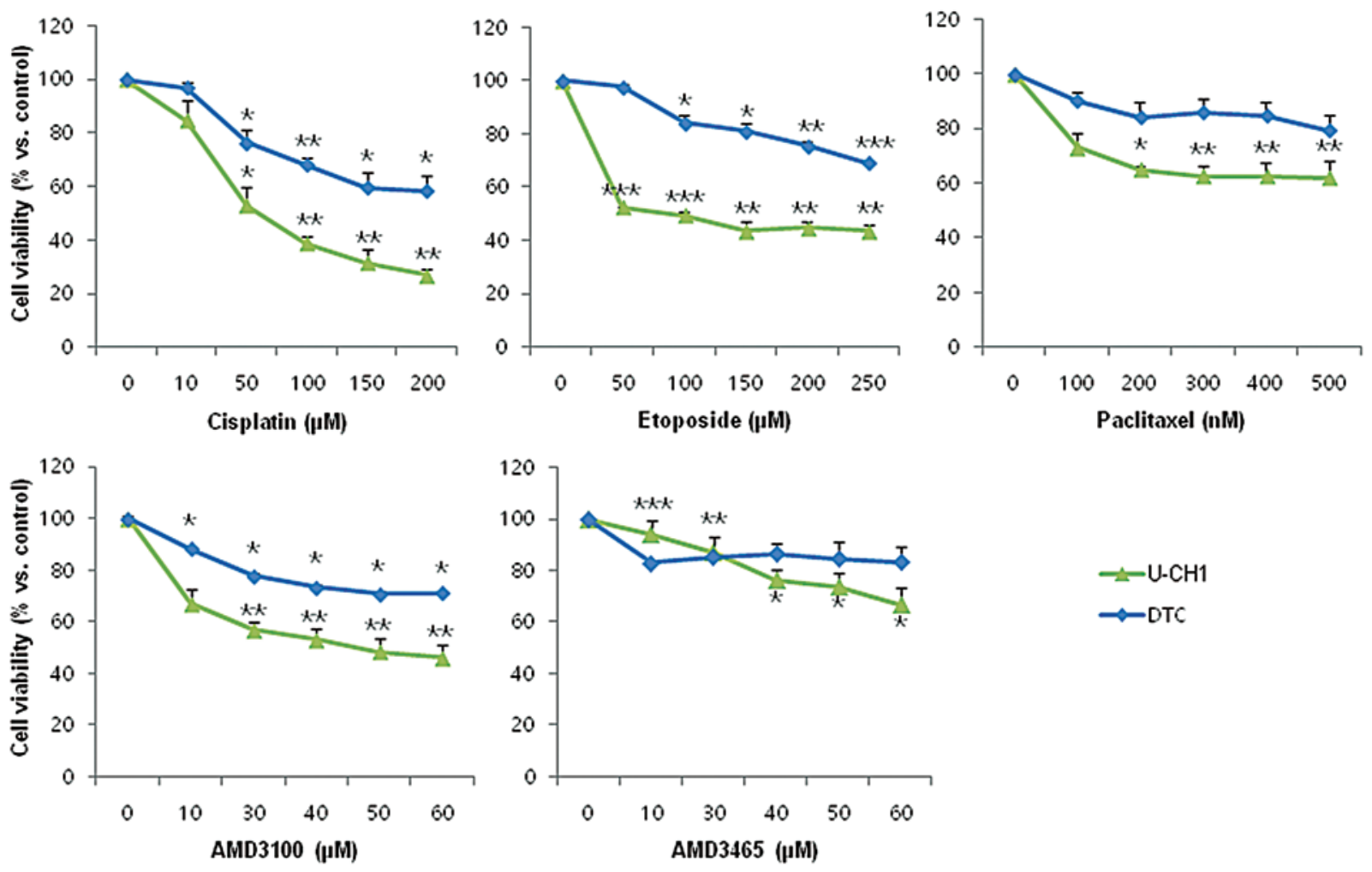

FIG. 6. Effect of drugs on the cytotoxicity of DTC and U-CH1 cells. MTS assay of DTC and U-CH1 cells treated with various concentrations of drugs. ${ }^{*} 0.01<p<0.05 ;{ }^{* *} 0.005<p<0.01 ;{ }^{* *} 0.001<p<0.005$. Figure is available in color online only. 


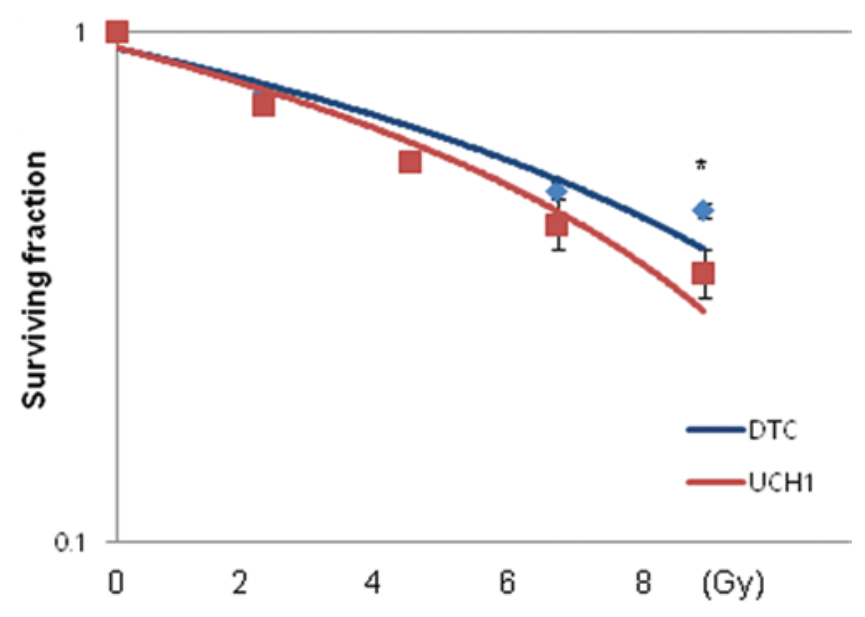

FIG. 7. Effect of IR on the cytotoxicity of DTC and U-CH1 cells. MTS assays of DTC and U-CH1 cells after 4 days of IR at various doses. *0.01 $<p<0.05$. Figure is available in color online only. possible therapeutic target to enhance the effectiveness of chemo- and radiotherapy of chordoma. In addition, the high tumorigenic potential of DTC cells in vivo and the fact that the pathological features of the xenograft tumor were similar to those of the patient tissue would make it possible to use these cells for developing suitable therapeutic strategies to treat patients with malignant chordoma.

Almost all chordomas express the notochord developmental transcription factor, brachyury, a crucial biomarker for chordoma. ${ }^{14}$ Along with brachyury, cytokeratin is also known as a combinational marker in chordoma. ${ }^{21}$ In addition, receptor tyrosine kinases, including PDGFR- $\alpha$ and $-\beta$, EGFR, and c-Met, are frequently expressed in chordomas, resulting in the activation of the downstream Akt and mTOR signaling pathways. ${ }^{1,2,20}$ Our Western blot and immunocytochemistry analyses indicated that the DTC cells also expressed brachyury, albeit at a much lower level than what was observed in U-CH1 cells. With regard to cytokeratins, the DTC cells showed significantly lower expression of cytokeratins 8 and 18 compared with the U-CH1 cells. DTC's reduced expression of cytokeratins and its
A
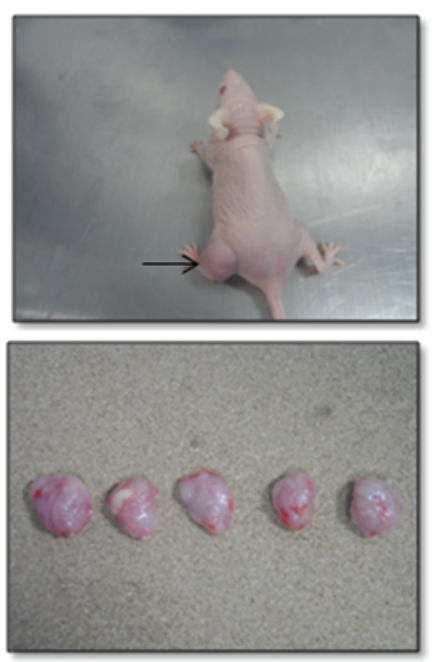

C
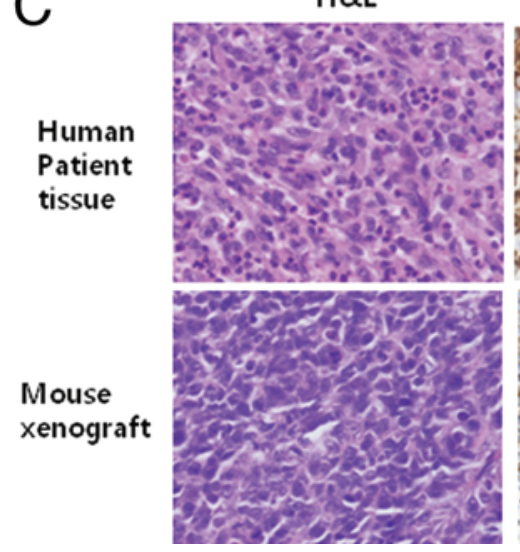
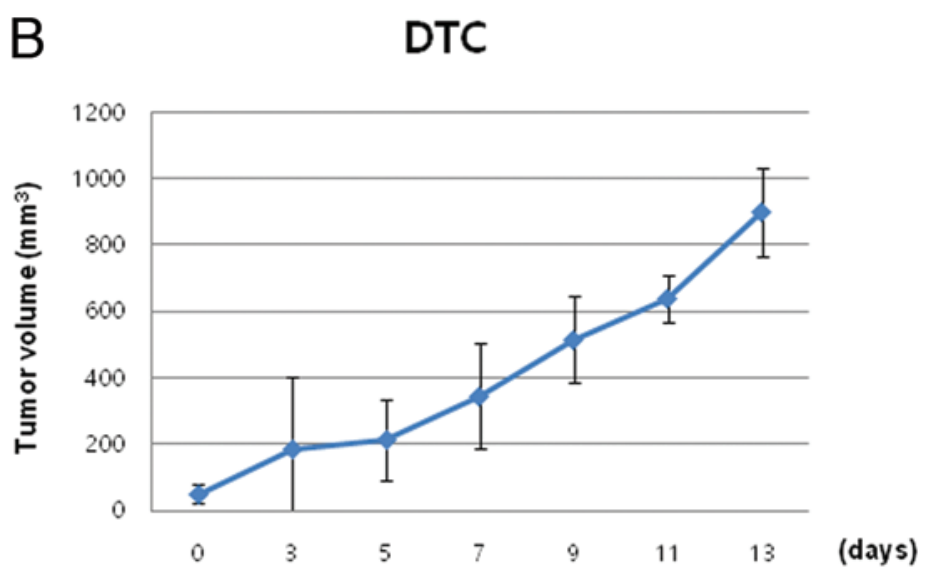

Cytokeratin

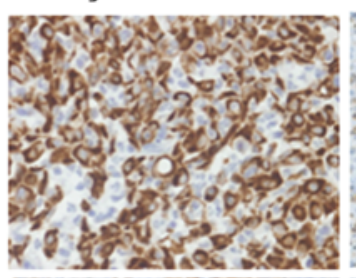

CXCR4
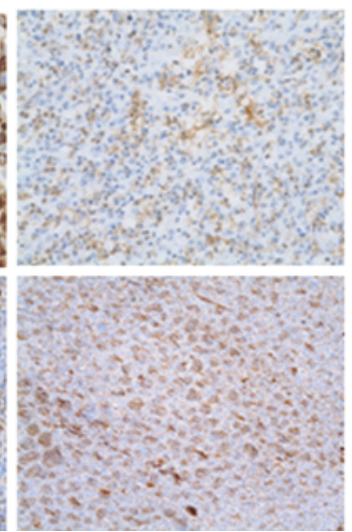

FIG. 8. In vivo tumor formation of DTC cells. A: Representative picture of the mouse (upper). Arrow indicates the site of the tumor. Tissue specimens isolated from mice (lower). B: Growth pattern of xenograft tumor. C: $\mathrm{H} \& \mathrm{E}$ and immunostaining of tissue specimens from a human patient with DTC (upper) and the mouse xenograft of DTC cells (lower). Both tumors showed similar morphology with slightly decreased cytokeratin and CXCR4 expression in the xenograft tumor. Original magnification $\times 400$. Figure is available in color online only. 
morphological characteristics are reminiscent of the EMT features. Indeed, the DTC cells showed enhanced expression of EMT-associated proteins including N-cadherin, Slug, and Twist. They also exhibited lower PDGFR- $\alpha$ and higher PDGFR- $\beta$ expressions than the U-CH1 cells. In line with these results, the phosphorylation of signaling molecules including p-ERK, p-Akt, p-mTOR, and p-STAT3, but not p-JNK, was much weaker in the DTC cells than in the U-CH1 cells. The DTC cells did not express EGFR and CD44, as determined by Western blot and FACS analyses. c-Met expression was similar in both cell lines. Interestingly, although total expression of CD24 was nearly the same in both cell lines, a cell surface level of CD24 was only detected in U-CH1 cells. Further study will be needed to define the meaning and mechanism of cell surface localization of CD24 in chordoma cells. Collectively, our data indicate that the established DTC cell line shows different expression pattern of chordoma markers compared with the conventional-type chordoma cell line and tissues.

One of the most interesting expression patterns of the DTC cells was their higher surface expression of CXCR4 compared with the U-CH1 cells, although the total expression of CXCR4 was indistinguishable between the two cell lines. Treatment with the CXCR4-specific inhibitor AMD3100 significantly suppressed the growth of both DTC and U-CH1 cells. Intriguingly, the U-CH1 cells were more sensitive to AMD3100 treatment than were the DTC cells. Because CXCR4 is known as a cancer stem cell marker in various cancers, ${ }^{6,10}$ we compared stem cell marker expression patterns between the two cell lines, finding that the DTC cells expressed higher levels of stem cell markers, including CD133, c-Myc, Oct4, and Musashi, than the U-CH1 cells. However, Notch1 and Sox 2 expression was similar in both cell lines, and Nanog was only expressed in the U-CH1 cells. Therefore, these data suggest that CXCR4 signaling along with enhanced stemness would be critical for the growth of DTC and U-CH1 cells. Accordingly, CXCR4 might be a good therapeutic target for treating malignant chordoma. In future studies we will further investigate the involvement of CXCR4 signaling in the stemlike cell characteristics of chordoma cells.

\section{Conclusions}

In the present study, we established a chordoma cell line from a patient with recurrent DTC. Compared with the U-CH1 cells, the established DTC cells exhibited lower expression of chordoma markers, brachyury and cytokeratins; higher expression of EMT- and stem cell-related markers; and higher surface expression of CXCR4. It has been established that the DTC cells are capable of producing tumors in nude mice. Moreover, the major differences in DTC cell line compared with previously established chordoma cell lines are that it is the first cell line raised from DTC and the cells exhibit high cell surface expression of CXCR4, which can be a molecular target for therapy in this type of tumor. Collectively, our data provide evidence indicating that the established DTC cells could be used to study the biology of DTCs in vitro and in vivo and to develop appropriated therapeutic targets in malignant chordoma.

\section{Acknowledgments}

We would like to give thanks to the Chordoma Foundation (Durham, NC) for providing a chordoma cell line, U-CH1. This study was supported by the national R\&D Program through the Korea Institute of Radiological and Medical Sciences funded by the Ministry of Science, ICT \& Future Planning (grants 1711021879 and 1711021925).

\section{References}

1. Akhavan-Sigari R, Abili M, Gaab MR, Rohde V, Zafar N, Emami P, et al: Immunohistochemical expression of receptor tyrosine kinase PDGFR- $\alpha$, c-Met, and EGFR in skull base chordoma. Neurosurg Rev 38:89-99, 2015

2. Akhavan-Sigari R, Gaab MR, Rohde V, Abili M, Ostertag H: Expression of PDGFR- $\alpha$, EGFR and c-MET in spinal chordoma: a series of 52 patients. Anticancer Res 34:623-630, 2014

3. Brüderlein S, Sommer JB, Meltzer PS, Li S, Osada T, Ng D, et al: Molecular characterization of putative chordoma cell lines. Sarcoma 2010:630129, 2010

4. Chugh R, Tawbi H, Lucas DR, Biermann JS, Schuetze SM, Baker LH: Chordoma: the nonsarcoma primary bone tumor. Oncologist 12:1344-1350, 2007

5. DeComas AM, Penfornis P, Harris MR, Meyer MS, Pochampally RR: Derivation and characterization of an extra-axial chordoma cell line (EACH-1) from a scapular tumor. J Bone Joint Surg Am 92:1231-1240, 2010

6. Gagliardi F, Narayanan A, Reni M, Franzin A, Mazza E, Boari N, et al: The role of CXCR4 in highly malignant human gliomas biology: current knowledge and future directions. Glia 62:1015-1023, 2014

7. Healey JH, Lane JM: Chordoma: a critical review of diagnosis and treatment. Orthop Clin North Am 20:417-426, 1989

8. Horiguchi H, Sano T, Qian ZR, Hirokawa M, Kagawa N, Yamaguchi T, et al: Expression of cell adhesion molecules in chordomas: an immunohistochemical study of 16 cases. Acta Neuropathol 107:91-96, 2004

9. Hsu W, Mohyeldin A, Shah SR, ap Rhys CM, Johnson LF, Sedora-Roman NI, et al: Generation of chordoma cell line JHC7 and the identification of Brachyury as a novel molecular target. J Neurosurg 115:760-769, 2011

10. Jung MJ, Rho JK, Kim YM, Jung JE, Jin YB, Ko YG, et al: Upregulation of CXCR4 is functionally crucial for maintenance of stemness in drug-resistant non-small cell lung cancer cells. Oncogene 32:209-221, 2013

11. Karikari IO, Gilchrist CL, Jing L, Alcorta DA, Chen J, Richardson WJ, et al: Molecular characterization of chordoma xenografts generated from a novel primary chordoma cell source and two chordoma cell lines. J Neurosurg Spine 21:386-393, 2014

12. Liu X, Nielsen GP, Rosenberg AE, Waterman PR, Yang W, Choy E, et al: Establishment and characterization of a novel chordoma cell line: CH22. J Orthop Res 30:1666-1673, 2012

13. McMaster ML, Goldstein AM, Bromley CM, Ishibe N, Parry DM: Chordoma: incidence and survival patterns in the United States, 1973-1995. Cancer Causes Control 12:1-11, 2001

14. Nibu Y, José-Edwards DS, Di Gregorio A: From notochord formation to hereditary chordoma: the many roles of brachyury. BioMed Res Int 2013:826435, 2013

15. Ostroumov E, Hunter CJ: The role of extracellular factors in human metastatic chordoma cell growth in vitro. Spine (Phila Pa 1976) 32:2957-2964, 2007

16. Presneau N, Shalaby A, Ye H, Pillay N, Halai D, Idowu B, et al: Role of the transcription factor $\mathrm{T}$ (brachyury) in the pathogenesis of sporadic chordoma: a genetic and functionalbased study. J Pathol 223:327-335, 2011 
17. Rinner B, Froehlich EV, Buerger K, Knausz H, Lohberger B, Scheipl S, et al: Establishment and detailed functional and molecular genetic characterisation of a novel sacral chordoma cell line, MUG-Chor1. Int J Oncol 40:443-451, 2012

18. Scheil S, Brüderlein S, Liehr T, Starke H, Herms J, Schulte $\mathrm{M}$, et al: Genome-wide analysis of sixteen chordomas by comparative genomic hybridization and cytogenetics of the first human chordoma cell line, U-CH1. Genes Chromosomes Cancer 32:203-211, 2001

19. Scheil-Bertram S, Kappler R, von Baer A, Hartwig E, Sarkar M, Serra M, et al: Molecular profiling of chordoma. Int J Oncol 44:1041-1055, 2014

20. Tamborini E, Virdis E, Negri T, Orsenigo M, Brich S, Conca $\mathrm{E}$, et al: Analysis of receptor tyrosine kinases (RTKs) and downstream pathways in chordomas. Neuro Oncol 12:776789, 2010

21. Yang C, Hornicek FJ, Wood KB, Schwab JH, Choy E, Iafrate J, et al: Characterization and analysis of human chordoma cell lines. Spine (Phila Pa 1976) 35:1257-1264, 2010

\section{Disclosures}

The authors report no conflict of interest concerning the materials or methods used in this study or the findings specified in this paper.

\section{Author Contributions}

Conception and design: Chang, Park. Acquisition of data: Kim, Lee, Koh. Analysis and interpretation of data: Chang, Koh, Park. Drafting the article: Chang, Park. Critically revising the article: Chang, Koh, Park. Reviewed submitted version of manuscript: all authors. Approved the final version of the manuscript on behalf of all authors: Chang. Administrative/technical/material support: Kim. Study supervision: Chang.

\section{Correspondence}

Ung-Kyu Chang, Department of Neurosurgery, Korea Institute of Radiological and Medical Sciences, Nowonro 75, Nowon-ku, Seoul 139-706, Korea. email: cuk@kcch.re.kr. 